\title{
Impacto de un programa de atención domiciliaria al enfermo crónico en ancianos: calidad de vida y reingresos hospitalarios
}

\author{
María Claudia Espinel-Bermúdez, OD, MsC, (I) Sergio Sánchez-García, CD, MsC, PhD, (I) Teresa Juárez-Cedillo, QFB, MsC,(I) \\ José Juan García-González, MD, MsC, (2) Álvaro Viveros-Pérez, BA, (I) Carmen García-Peña, MD, MsC, PhD.(I)
}

\author{
Espinel-Bermúdez MC, Sánchez-García S, Juárez-Cedillo T, \\ García-González JJ,Viveros-Pérez A, García-Peña C. \\ Impacto de un programa de atención domiciliaria \\ al enfermo crónico en ancianos: calidad de vida \\ y reingresos hospitalarios. \\ Salud Publica Mex 20I I;53:17-25.
}

\section{Resumen}

Objetivo. Evaluar el impacto del programa Atención Domiciliaria al Enfermo Crónico (ADEC) comparado con la atención habitual $(\mathrm{AH})$ a ancianos con dependencia funcional, derechohabientes del Instituto Mexicano del Seguro Social (IMSS). Material y métodos. Cohorte prospectiva a tres meses a partir del egreso hospitalario en dos hospitales de la Ciudad de México. Se ingresaron 130 ancianos con dependencia funcional, 70 insertados al programa ADEC y 60 con atención habitual. Se midió impacto en reingresos hospitalarios y calidad de vida a partir de la escala Perfil de Impacto de la Enfermedad (SIP, por sus siglas en inglés). Resultados. La edad promedio de los ancianos fue de 74 años $(61 / 103)$ y $60 \%$ fueron mujeres. El principal diagnóstico fue enfermedad vascular cerebral (EVC) (30.77\%). El grupo de ADEC mejoró la calidad de vida en la dimensión psicosocial [46.26 ( \pm |3.85) comparado con 29.45 ( \pm 16.48$)$ vs. $47.03( \pm 16.47)$ a $42.36( \pm 16.35) p<0.05$ en grupo habitual]. No se presentaron diferencias en los reingresos $(p>0.05)$. Conclusiones. El programa mejoró la dimensión psicosocial de calidad de vida.

Palabras clave: servicios de atención de salud a domicilio; anciano; evaluación de procesos y resultados (atención de salud); México
Espinel-Bermúdez MC, Sánchez-García S, Juárez-Cedillo T, García-González JJ,Viveros-Pérez A, García-Peña C.

Impact of the program home care

for the chronically ill for elderly: quality

of life and hospital readmissions.

Salud Publica Mex 20II;53:17-25.

\begin{abstract}
Objective. To evaluate the impact of the ADEC program (acronym in Spanish) as compared with the typical care provided to disabled elderly affiliated with the Mexican Institute of Social Security (IMSS). Material and Methods. Prospective cohort at three months after discharge from two general hospitals in Mexico City. A total of I 30 patients with functional dependency were studied, 70 in the ADEC program and 60 with typical care. Impact was measured using hospital readmissions and quality of life based on the Sickness Impact Profile (SIP). Results. Average age was 74 $(61 / / 03)$ years and $60 \%$ were women. The main diagnosis was cerebrovascular disease $(30.77 \%)$. The quality of life in the psychosocial dimension improved for the ADEC group (from $46.26( \pm 13.85)$ to $29.45( \pm 16.48)$ as compared with $47.03( \pm 16.47)$ to $42.36( \pm 16.35)$ for those receiving typical care $(p<0.05)$. No differences were found regarding hospital readmissions. ( $p>0.05)$. Conclusions. HC program improved the psychosocial dimension of quality of life.
\end{abstract}

Key words: home care services, aged, outcome and process assessment (health care); Mexico

(I) Unidad de Investigación Epidemiológica y en Servicios de Salud, Área Envejecimiento, Instituto Mexicano del Seguro Social. México, DF.

(2) Hospital General Regional N ${ }^{\circ}$, Instituto Mexicano del Seguro Social. Querétaro, Querétaro.

Fecha de recibido: 27 de julio de 2010 - Fecha de aceptado: 13 de octubre de 2010

Solicitud de sobretiros: Dra. Carmen García-Peña. Unidad de Investigación Epidemiológica y en Servicios de Salud, Área Envejecimiento, Instituto Mexicano del Seguro Social, Centro Médico Siglo XXI. Av. Cuauhtémoc 330, Col. Doctores. 06725 México DF, México. Correo electrónico: carmen.garcia@imss.gob.mx,mcgarciapena@gmail.com 
E número de ancianos que requieren cuidados a largo plazo ha aumentado considerablemente ante la presencia de enfermedades crónicas y discapacidad, situaciones que representan un reto importante para los servicios de salud ya que su atención continúa aún después del egreso hospitalario. ${ }^{1-3}$

La inserción de programas de atención domiciliaria posterior al egreso hospitalario podría representar una alternativa viable al combinar la atención en salud al anciano con esquemas de monitoreo y educación a pacientes y cuidadores, que permiten proporcionar cuidados a largo plazo en el hogar de los ancianos con la atención de equipos médicos y / o paramédicos. Estudios previos han evaluado la efectividad e impacto de estos esquemas en diferentes grupos con gran heterogeneidad de esquemas y efectos encontrados. ${ }^{4}$ Una revisión sistemática que incluyó 15 diferentes estudios ${ }^{5}$ demostró que esquemas basados en actividades de prevención y promoción de la salud reducían significativamente el riesgo de mortalidad de los ancianos así como la admisión a instituciones de atención a largo plazo, a pesar de que no se logró reducir el número de reingresos hospitalarios o la dependencia en las actividades de la vida diaria de los ancianos. Asimismo, cuando se insertaron ancianos a esquemas de atención domiciliaria presentaron mayores reingresos hospitalarios $(17 \%, p=$ 0,012 ) a tres meses de seguimiento en comparación con los ancianos que recibieron atención hospitalaria. ${ }^{6}$

Otros estudios han demostrado cambios en la calidad de vida ${ }^{6}$ en ancianos con enfermedades terminales insertados a un esquema de atención domiciliaria; al analizar la calidad de vida por dimensiones, se encontraron resultados a favor de la atención domiciliaria en aspectos como rol emocional, función social y salud mental, mientras que el grupo control reportó mayor dolor corporal. ${ }^{7}$ Se ha concluido que la calidad de vida podría representar un impacto sensible en las actividades de este esquema de atención y que los efectos positivos se relacionan con el mejoramiento de las condiciones de vida del anciano y la integración con su comunidad. ${ }^{8}$

En México, las instituciones públicas de salud cuentan con escasos ejemplos de esquemas de atención domiciliaria, entre los que destaca el programa del gobierno del Distrito Federal a población abierta. Por su parte, el Instituto Mexicano del Seguro Social (IMSS) ofrece desde 1991 atención a través del Programa de Atención Domiciliaria al Enfermo Crónico (ADEC) a partir del egreso hospitalario en el segundo nivel de atención, y aunque cubre a población de 18 años en adelante, $80 \%$ de sus servicios se enfocan a la atención de ancianos, sin embargo, a la fecha no se ha evaluado el impacto de sus acciones en esta población pues los resultados previos han considerado el total de población atendida así como diversos impactos. ${ }^{9-12}$

En este sentido, el objetivo de este estudio es evaluar el impacto del programa de atención domiciliaria comparado con la atención habitual otorgada a ancianos con dependencia funcional, derechohabientes del Instituto Mexicano del Seguro Social.

\section{Material y métodos}

Estudio de cohorte prospectivo con seguimiento a tres meses. La población de estudio estuvo conformada por ancianos de 60 y más años con dependencia funcional, hospitalizados en dos hospitales del IMSS ubicados en el área metropolitana de la Ciudad de México. La medición inicial se realizó entre el 22 de agosto del 2006 y el 29 de junio de 2007 y se siguieron a los pacientes por un lapso de tres meses, desde el ingreso al programa hasta el alta del mismo (tres meses después).

Un hospital contaba con el programa de atención domiciliaria al enfermo crónico (ADEC) (grupo de estudio); el otro hospital (grupo de comparación) no lo tenía y sólo contaba con esquemas de atención habitual (hospitalización y remisión a servicios de primer nivel según necesidad). Ambos hospitales fueron considerados hospitales generales de mediana complejidad; el número de camas disponibles en el estudio fue de 258 camas y en el de comparación de 319. Ambos contaban con los servicios básicos de urgencias, medicina interna, cirugía, y pediatría.

Criterios de selección al estudio. Se consideraron ancianos de 60 años en adelante, seleccionados por los integrantes del programa en las áreas de hospitalización de los servicios de medicina interna y cirugía, y que al egreso hospitalario se insertaban por primera vez al programa ADEC. Para el grupo de atención habitual se seleccionaron pacientes con mismo diagnóstico de funcionalidad que en el grupo ADEC. Se excluyeron ancianos que recibieron atención hospitalaria en fechas diferentes al periodo de estudio, que fueron referidos al programa ADEC del servicio de urgencias, de clínicas de medicina familiar $u$ hospitales de alta especialidad, y que no se encontraban hospitalizados al momento de la evaluación inicial.

Tamaño de la muestra. Se definió a partir de la fórmula para comparar dos proporciones con los siguientes supuestos: alfa de 0.05 (dos colas), poder de $80 \%$, proporción de reingresos hospitalarios de acuerdo a estudio previo 9 en el que para grupo de atención domiciliaria la proporción fue de 0.45 y para grupo de atención habitual 0.23 . Además se adicionó un $25 \%$ por pérdidas durante el seguimiento, por lo que quedó conformado 
cada grupo por 80 ancianos. Se realizó muestreo por cuota y a conveniencia de acuerdo con los ingresos de pacientes del programa ADEC.

\section{Periodo de análisis}

La medición inicial se realizó entre el 22 de agosto de 2006 y el 29 de junio de 2007, y se siguió a los pacientes por un lapso de tres meses desde el ingreso al programa hasta el alta del mismo. Se estableció como tiempo cero del estudio el día de egreso hospitalario de los sujetos en el grupo de estudio (insertado al programa ADEC), momento en que iniciaba la intervención del programa.

\section{Descripción del programa de atención domiciliaria ADEC}

Los pacientes hospitalizados fueron identificados como elegibles conforme a procedimientos habituales del programa, ${ }^{13}$ basados en criterios de inclusión del mismo y sin injerencia de los investigadores. El programa señala que deberán incluirse pacientes con alguna de las siguientes condiciones: polipatología y pérdida de la autonomía permanente o temporal, enfermedad neurológica con pérdida total de la autonomía permanente y / o traqueotomía, pérdida sensorial reciente que limite la funcionalidad en forma temporal para adaptación al medio, enfermedad aguda o crónica que comprometa la autonomía en forma temporal o transitoria, demencia en etapas avanzada y post-operado de cirugía mayor y con pérdida de la autonomía temporal. Los pacientes debían contar con cuidador y/o red de apoyo familiar o comunitaria, ingresar al programa en forma voluntaria y vivir en el área de influencia del hospital. El equipo realizó también una evaluación médico-social que se aplicó 72 horas previas al egreso hospitalario; posteriormente se realizó una sesión educativa dirigida a informar a la familia sobre el estado de salud del anciano y las necesidades de cuidado que tendría en casa. A partir del egreso hospitalario iniciaron las actividades del programa con una segunda sesión educativa la semana siguiente del egreso; esta sesión se realiza en conjunto con otras familias de pacientes que han sido dados de alta y tiene por objeto concientizar a la familia sobre el cuidado a largo plazo que requiere un paciente y a la red de apoyo necesaria para proveerle atención domiciliaria. Posteriormente se programan las visitas a domicilio. Además, durante el tiempo que el anciano estuvo insertado al programa (3 meses) se le proporcionó material médico quirúrgico, medicamentos y alimentación complementaria, y se contó con una línea telefónica a la cual podían llamar los familiares cuando requirieran información o cuando detectaban que su familiar se encontraba enfermo.

El programa de atención domiciliaria se constituye por dos equipos de salud, uno fijo en la unidad hospitalaria y otro móvil que realiza las visitas domiciliarias; cada equipo está conformado por un médico, una enfermera, una psicóloga y un residente de medicina interna. Las visitas a domicilio incluyen revisión de la vivienda para dar recomendaciones que mejoren la estancia del anciano, evaluaciones médicas al pacientee instrucción al cuidador en cuanto al manejo básico de la enfermedad y la detección de signos de alarma. Durante los tres meses de seguimiento, cada anciano recibió dos visitas del equipo médico en su domicilio.

Mientras tanto, el grupo control no recibió visitas de atención domiciliaria; solamente recibió la atención habitual que ofreció la red de servicios del IMSS.

\section{Mediciones}

La evaluación basal fue realizada simultáneamente en cada grupo. El día del egreso hospitalario de los sujetos que iban a ser insertados al programa, se recabó información en un cuestionario elaborado ex profeso para el estudio en el que se incluyeron preguntas relativas a variables sociodemográficas, uso previo de servicios, que incluyen admisiones hospitalarias previas, diagnóstico(s) principal(es) de ingreso a la hospitalización actual y escala abreviada del estado mental (MMSE) adaptada a ancianos mexicanos. ${ }^{14}$ Se midió funcionalidad con el índice de Katz, ${ }^{15}$ que es una escala de valoración de la función física que pretende medir la autonomía y dependencia del sujeto para realizar actividades de la vida diaria. Concretamente, el índice de Katz valora la posibilidad de higiene, vestimenta, movilidad, continencia y alimentación. Según el índice, se considera independiente al sujeto que no necesita ayuda de otra persona o de algún accesorio (bastón, andadera etc). Las preguntas son jerarquizadas y deben ser realizadas directamente al sujeto en compañía de sus cuidadores, si es que los tiene.

La calidad de vida se midió aplicando el Sickness Impact Profile, ${ }^{16}$ adaptado en México ${ }^{17}$ y validado al español. ${ }^{18}$ Este es un instrumento diseñado para registrar cambios en la calidad de vida de los pacientes y determinar el impacto de la enfermedad en la vida diaria. El cuestionario consta de 136 ítems agrupados en 12 categorías diferentes. Tres de esas categorías (cuidado personal, movilidad y deambulación) comprenden la dimensión física (dimensión I), mientras que cuatro de las categorías (estado emocional, interacción social, estado de alerta y comunicación) comprenden la di- 
mensión psicosocial (dimensión II). Las restantes cinco categorías (sueño y descanso, labores del hogar, trabajo, recreaciones, pasatiempos y alimentación) constituyen la dimensión de categorías independientes (dimensión III). Los ítems incluidos en el cuestionario describen conductas o actividades que pueden o no relacionarse con su estado de salud. Los pacientes indicaron cuáles ítems los describían personalmente y el puntaje se calculó para cada categoría al sumar cada ítem con respuesta afirmativa y al dividir entre el máximo posible de disfunción. Se agruparon los puntajes de cada categoría y se multiplicaron por 100 para tener un puntaje total de 0, para el mínimo impacto de la enfermedad sobre su calidad de vida, y 100, para el máximo impacto.

La comorbilidad fue medida con el Índice de Charlson. ${ }^{19}$ Este índice predice la mortalidad a un año para un paciente que puede tener un abanico de condiciones comórbidas como insuficiencia cardiaca, enfermedad respiratoria crónica, diabetes, hemiplejia, insuficiencia renal crónica, sida o cáncer, hasta un total de 22 condiciones. A cada condición se le asigna una puntuación de 1, 2, 3 o 6 dependiendo del riesgo de fallecer asociado a esta condición. Las puntuaciones totales permiten establecer un rango de morbilidad alto, medio o bajo.

Se hicieron también mediciones de uso de servicios en los seis meses previos a la hospitalización y de variables sociodemográficas.

Tres meses después de iniciadas las actividades del programa, se realizó una segunda medición a participantes de cada grupo que incluyó las mismas variables de la medición basal y de la evaluación de calidad de vida de los ancianos, además de reingresos hospitalarios que se definieron como toda admisión a urgencias o a servicios hospitalarios motivada por la misma condición por la cual fue egresado. Esta información fue solicitada a los sujetos en una serie de preguntas elaboradas ex profeso para medir esta variable. Para confirmar la información proporcionada, todas las admisiones realizadas a los hospitales de segundo nivel de referencia fueron cotejadas contra los expedientes de los pacientes.

\section{Análisis estadístico}

Se analizaron las características generales y clínicas de los participantes de cada grupo a fin de establecer homogeneidad en las variables al tiempo cero. Se realizó análisis descriptivo a partir de frecuencias simples y relativas, medidas de tendencia central y de dispersión. Las variables de estudio fueron contrastadas entre el tiempo cero ( $\mathrm{t} 0$ ) y tiempo uno (t1), como muestras relacionadas a partir de prueba de Wilcoxon para el análisis de calidad de vida entre las mediciones basal y final de cada grupo y como muestras independientes con prueba de U de Mann-Whitney, para el análisis de los reingresos hospitalarios entre los dos grupos. Por último, se realizaron análisis bivariados y multivariados para identificar variables con asociación significativa al resultado de reingresos y calidad de vida en sus diferentes dimensiones. Se utilizó software SPSS 16 y STATA SE 8.

Finalmente, se solicitó consentimiento informado por escrito a los participantes en el estudio y a sus cuidadores, con fundamento en los preceptos éticos nacionales e internacionales. En caso de que los ancianos se encontraran limitados para dar su consentimiento informado, se obtuvo autorización de su cuidador principal o familiar responsable. El proyecto fue aprobado por el Comité Local de Investigación con el número 2005-3607-0016.

\section{Resultados}

Se incluyeron 70 ancianos y sus cuidadores que recibieron beneficios del programa de atención domiciliaria, y 60 ancianos y sus cuidadores con atención habitual. El cuadro I presenta características demográficas de los ancianos por grupos en línea basal; la mediana de edad fue de 75 años (61/103), similar entre grupos, $60 \%$ fueron mujeres, $55.38 \%$ no tenían pareja, y no se reportaron diferencias en ingresos económicos mensuales. En cuanto al grado de escolaridad, sólo $12.86 \%$ de los sujetos en el grupo de estudio reportó no tener escolaridad, frente a $36.67 \%$ del grupo de comparación ( $p>0.05$ ).

En cuanto a las variables clínicas, el principal diagnóstico en los dos grupos fue enfermedad vascular cerebral $(30.77 \%)$, seguido de insuficiencia renal crónica $(14.6 \%)$. Se encontraron diferencias significativas $(p<0.05)$ en el tiempo de estancia con 16 días (2/64) en el grupo de ADEC y 33 días en el grupo de atención habitual (5/254). Se presentó una alta comorbilidad en $61.43 \%$ de los sujetos del grupo de estudio, en contraste con $46.67 \%$ de los sujetos del grupo de comparación que no presentaron comorbilidad agregada $(p<0.05)$ (cuadro I).

En cuanto a las variables evaluadas en la basal, se encontró que los reingresos previos no presentaron diferencias significativas entre los grupos. De igual manera se evidenció que no hubo diferencias significativas en el puntaje global de calidad de vida a excepción de la dimensión física, en la cual los ancianos del grupo de intervención reportaron una mejor calidad de vida en cuanto a la dimensión física (47.60土11.20) en contraste con el grupo de comparación que obtuvo un puntaje mayor $(52.89 \pm 16.12)$

Al término del seguimiento la tasa de defunción para el grupo ADEC fue 6.5 (45 decesos) y para el gru- 
Cuadro I

\section{Características basales de la población en estudio. México DF, Junio 2006-junio 2007}

$\begin{array}{ccc}\text { ADEC } & \text { Atención habitual } & \text { Total } \\ n=70 & n=60 & n=130 \\ n(\%) & n(\%) & n(\%)\end{array}$

I.Variables sociodemográficas

\begin{tabular}{|c|c|c|c|c|}
\hline Sexo & & & & \\
\hline Masculino & $23(32.86)$ & $29(48.33)$ & $52(40.00)$ & \\
\hline Femenino & $47(67.14)$ & $3 I(5 I .67)$ & $78(60.00)$ & 0.071 \\
\hline \multicolumn{5}{|l|}{ Edad (años) } \\
\hline Mediana & 76 & 76 & 75 & 0.876 \\
\hline Min/máx. & $61-103$ & $61-102$ & $61-103$ & \\
\hline Escolaridad & & & & $0.003^{*}$ \\
\hline Ninguna & $9(12.86)$ & $22(36.67)$ & $31(23.85)$ & \\
\hline Básica & $50(71.43)$ & $36(60.00)$ & $86(66.15)$ & \\
\hline Medio superior & $7(10.00)$ & $2(3.33)$ & $9(6.92)$ & \\
\hline Superior & $4(5.7 I)$ & $0(0.00)$ & $4(3.08)$ & \\
\hline Apoyos económicos & & & & 0.274 \\
\hline Sin apoyo económico & $28(40.00)$ & $20(33.33)$ & $48(36.92)$ & \\
\hline Con apoyo económico & $42(60.00)$ & $40(66.67)$ & $82(63.08)$ & \\
\hline $\begin{array}{l}\text { Apoyo mensual } \\
\text { Media }( \pm D E)\end{array}$ & $1622.6( \pm 1878.06)$ & $\mid 809.7( \pm|| 6|.3|)$ & $17 \mid 3.88( \pm 1563.18)$ & 0.720 \\
\hline Min/máx. & $200 / 10000$ & $100 / 4500$ & $100 / 10000$ & \\
\hline Red de apoyo & & & & 0.089 \\
\hline Baja red de apoyo & $33(47.14)$ & $18(30.00)$ & $51(39.23)$ & \\
\hline Mediana red de apoyo & $18(25.72)$ & $21(35.00)$ & $39(30.00)$ & \\
\hline Alta red de apoyo & $19(27.14)$ & $21(35.00)$ & $40(30.77)$ & \\
\hline
\end{tabular}

II.Variables clínicas

Diagnóstico principal de ingreso

0.987

Enfermedad vascular cerebral

\begin{tabular}{rrr}
$20(28.57)$ & $20(33.33)$ & $40(30.77)$ \\
\hline $11(15.71)$ & $8(13.33)$ & $19(14.62)$ \\
\hline $10(I 4.29)$ & $5(8.33)$ & $15(I 1.53)$ \\
\hline $29(41.43)$ & $27(45.01)$ & $56(43.08)$
\end{tabular}

$\begin{array}{lrrr}\text { Cáncer intraabdominal } & 10(14.29) & 5(8.33) & 15(\mid \mathrm{I} .53) \\ \text { Otros diagnósticos } & 29(41.43) & 27(45.01) & 56(43.08)\end{array}$

Días estancia hospitalaria

$0.007^{*}$

Mediana

$\begin{array}{rrr}16 & 33 & 24 \\ 2 / 64 & 5 / 254 & 2 / 254\end{array}$

Min/máx.

16

Comorbilidad (índice de Charlson)

Ausencia de comorbilidad

$14(20.00)$

$13(18.57)$

$43(61.43)$

$10(16.67)$

$42(32.31)$

Alta comorbilidad

23(17.69)

$65(50.00)$

ADEC:Atención Domiciliaria del Enfermo Crónico 
po de atención habitual 4.3 (23 decesos). Además, siete participantes (uno en el grupo ADEC y seis en el grupo de atención habitual) no pudieron ser localizados.

En relación con las variables finales, no se encontraron diferencias estadísticamente significativas entre los grupos en el número de reingresos, en este sentido, el tiempo al reingreso fue mayor en el grupo de ADEC con 25 (3/74) días, en contraste con 18 (1/69) días del grupo de atención habitual. Los días estancia del grupo de ADEC fueron 9 (1/78) días contra $12(1 / 80)$ en el grupo de atención habitual, sin presentar diferencias estadísticamente significativas, pero se consideró que desde el punto de vista clínico es relevante que los ancianos puedan retrasar el ingreso hospitalario y, aún más, los días de estancia hospitalaria (cuadro II).

La calidad de vida se evaluó en los sujetos sobrevivientes de cada grupo (24 del grupo de estudio y
31 del de comparación). El grupo de estudio mejoró significativamente la calidad de vida en la dimensión psicosocial al pasar de $46.26( \pm 13.85)$ a $29.45( \pm 16.48)$ vs. $47.03( \pm 16.47)$ a $42.36( \pm 16.35)$ (cuadro III).

A pesar de las diferencias estadísticamente significativas entre grupos en la fase basal de las variables sexo, escolaridad y comorbilidad, no se encontró asociación significativa con las variables de impacto al final del estudio. En el análisis bivariado para reingresos, se encontraron los siguientes resultados: sexo $p=0.605$ y escolaridad $p=0.545$; respecto al modelo para calidad de vida, la variable sexo tuvo un valor de significancia de 0.938 , escolaridad 0.083 y comorbilidad 0.316 . El modelo ajustado sólo aportaba $14 \%$ de la relación entre los grupos de estudio y las variables de impacto, por lo cual se presentan únicamente las tablas crudas que expresan los mismos resultados que los ajustados.

\section{Reingresos al tiempo uno ( $t_{1}$ ) entre los grupos de comparación. MéXico DF, junio 2006-junio 2007}

\begin{tabular}{|c|c|c|c|c|}
\hline & $\begin{array}{l}A D E C \\
n=69 \\
n(\%)\end{array}$ & $\begin{array}{c}\text { Atención habitual } \\
n=54 \\
n(\%)\end{array}$ & $\begin{array}{c}\text { Total } \\
n=123 \\
n(\%)\end{array}$ & $p$ \\
\hline Reingresos & & & & 0.523 \\
\hline No & $34(49.3)$ & $26(48.1)$ & $60(48.8)$ & \\
\hline Sí & $35(50.7)$ & $28(51.9)$ & $63(51.2)$ & \\
\hline Tipo de reingreso & $n=35$ & $n=28$ & $n=63$ & \\
\hline Urgencias & $\mathrm{II}(3 \mathrm{I} .43)$ & $8(28.57)$ & $19(30.16)$ & $0.033^{*}$ \\
\hline Hospitalización & $0(0.00)$ & $4(14.29)$ & $4(6.35)$ & \\
\hline Urgencias y hospitalización & $24(68.57)$ & $16(57.14)$ & $40(63.49)$ & \\
\hline Número de reingresos & & & & 0.558 \\
\hline I & $30(85.7)$ & $21(75.0)$ & $51(81.0)$ & \\
\hline 2 & $3(8.6)$ & $4(14.29)$ & $7(11.1)$ & \\
\hline 3 & $2(5.7)$ & $3(|0.7|)$ & $5(7.9)$ & \\
\hline Relación Dx reingreso con Dx principal & & & & 0.257 \\
\hline Sí & $15(42.9)$ & 16(57.1) & $31(49.2)$ & \\
\hline No & $18(51.4)$ & 12(42.9) & $30(47.6)$ & \\
\hline Sin información & $2(5.7)$ & $0(0.00)$ & $2(3.2)$ & \\
\hline Tiempo al Ier reingreso(días) & $n=23$ & $n=25$ & $n=48$ & 0.082 \\
\hline Mediana & 25 & 18 & 21 & \\
\hline $\operatorname{Min} / \max$ & $3 / 74$ & $1 / 69$ & $1 / 74$ & \\
\hline Estancia ler reingreso(días) & & & & 0.528 \\
\hline Mediana & 9 & 12 & 10 & \\
\hline $\operatorname{Min} / \max$ & $\mathrm{I} / 78$ & $1 / 80$ & $1 / 80$ & \\
\hline
\end{tabular}

ADEC:Atención Domiciliaria del Enfermo Crónico 


\section{Cuadro III}

Cambio en la calidad de vida de los sujetos vivos entre grupos de comparación.

MÉxico DF, JUNIO 2006-jUNIO 2007

\begin{tabular}{|c|c|c|c|c|c|c|c|c|c|c|}
\hline & \multicolumn{4}{|c|}{$\begin{array}{l}A D E C \\
n=24\end{array}$} & \multicolumn{4}{|c|}{$\begin{array}{c}\text { Atención habitual } \\
\qquad n=31\end{array}$} & \multicolumn{2}{|c|}{$\Delta t_{1} t_{l}$} \\
\hline & $t_{0}$ & $t_{1}$ & $\Delta t_{0} t_{1}$ & $P$ & $t_{0}$ & $t_{l}$ & $\Delta t_{0} t_{l}$ & $p$ & $\Delta t_{1} t_{l}$ & $p$ \\
\hline \multicolumn{11}{|l|}{ SIP Global } \\
\hline $\begin{array}{l}\text { Media } \\
( \pm \mathrm{DE})\end{array}$ & $\begin{array}{c}47.50 \\
( \pm 8.32)\end{array}$ & $\begin{array}{c}39.63 \\
( \pm 12.05)\end{array}$ & $\begin{array}{c}7.87 \\
( \pm 12.25)\end{array}$ & $0.005 *$ & $\begin{array}{c}49.27 \\
( \pm 11.34)\end{array}$ & $\begin{array}{c}44.99 \\
( \pm 12.05)\end{array}$ & $\begin{array}{c}4.28 \\
( \pm 15.02)\end{array}$ & 0.124 & $\begin{array}{l}-5.36 \\
( \pm 3.82)\end{array}$ & 0.153 \\
\hline \multicolumn{11}{|l|}{ Dimensiones } \\
\hline $\begin{array}{l}\text { Física } \\
\begin{array}{l}\text { Media } \\
( \pm D E)\end{array}\end{array}$ & $\begin{array}{c}47.23 \\
( \pm 10.30)\end{array}$ & $\begin{array}{c}48.74 \\
( \pm 12.15)\end{array}$ & $\begin{array}{c}-1.50 \\
( \pm I I .6 I)\end{array}$ & 0.532 & $\begin{array}{c}51.18 \\
( \pm 15.11)\end{array}$ & $\begin{array}{c}49.37 \\
( \pm 21.70)\end{array}$ & $1.81( \pm \mid 8.32)$ & 0.586 & $\begin{array}{c}-0.63 \\
( \pm 4.62)\end{array}$ & 0.892 \\
\hline $\begin{array}{c}\text { Psicosocial } \\
\text { Media } \\
( \pm D E)\end{array}$ & $\begin{array}{c}46.26 \\
( \pm \mid 3.85)\end{array}$ & $\begin{array}{c}29.45 \\
( \pm 16.48)\end{array}$ & $\begin{array}{c}16.80 \\
( \pm 21.16)\end{array}$ & $0.001 *$ & $\begin{array}{c}47.03 \\
( \pm 16.47)\end{array}$ & $\begin{array}{c}42.36 \\
( \pm 16.35)\end{array}$ & $\begin{array}{c}4.67 \\
( \pm 19.00)\end{array}$ & 0.182 & $\begin{array}{l}-12.90 \\
( \pm 4.46)\end{array}$ & $0.006^{\ddagger}$ \\
\hline $\begin{array}{c}\text { Independencia } \\
\text { Media } \\
( \pm \mathrm{DE})\end{array}$ & $\begin{array}{c}52.33 \\
( \pm \mid I .44)\end{array}$ & $\begin{array}{c}43.82 \\
( \pm 15.14)\end{array}$ & $\begin{array}{c}8.51 \\
( \pm 16.36)\end{array}$ & $0.018^{*}$ & $\begin{array}{l}50.46 \\
( \pm 17.63)\end{array}$ & $\begin{array}{c}39.76 \\
( \pm 16.03)\end{array}$ & $\begin{array}{c}10.70 \\
( \pm 19.58)\end{array}$ & $0.005^{*}$ & $\begin{array}{c}4.06 \\
( \pm 4.26)\end{array}$ & 0.345 \\
\hline
\end{tabular}

ADEC:Atención Domiciliaria del Enfermo Crónico

SIP: Sickness Impact Profile

* Cambio intragrupos estadísticamente significativo $(p<0.05)$ entre $t_{0}$ y $t_{1}$

‡ Cambio entregrupos estadísticamente significativo $(p<0.05)$ entre $\mathrm{t}_{1}$ y $\mathrm{t}_{1}$

\section{Discusión}

A pesar de que el envejecimiento puede ser entendido como el éxito de las políticas públicas y sociales a nivel mundial, al mismo tiempo se convierte en el principal reto para los servicios de salud, toda vez que los cambios demográficos cursan concurrentemente con cambios epidemiológicos que evidencian la necesidad de incorporar estrategias de atención a largo plazo que contengan los efectos de las enfermedades crónicas y la dependencia funcional de los ancianos. En este estudio se evaluó el impacto a tres meses de un esquema institucional de atención domiciliaria a ancianos de 60 y más años, en contraste con la atención habitual que reciben los pacientes al egreso hospitalario. En este sentido vale la pena considerar que se realizó una evaluación bajo las condiciones habituales de operación de un servicio, lo cual impide mantener el control de algunas variables.

Al término del seguimiento no se encontraron diferencias significativas en la readmisión hospitalaria, sin embargo, retrasar clínicamente el reingreso hospitalario y disminuir el tiempo de estancia de los pacientes podría explicarse por la influencia del programa que permite contener el uso de servicios y diferir esta atención al equipo de visitas domiciliarias.
Smith y colaboradores ${ }^{20}$ expresaron que el comportamiento de uso de servicios entre ancianos usuarios de atención domiciliaria es poco conocido. Estos investigadores realizaron un estudio de cohorte con seguimiento por ocho meses para analizar la proporción de uso de servicios hospitalarios en relación con la comorbilidad como potencial factor de reingreso hospitalario. Los autores encontraron que se presentó una proporción significativamente más alta en los reingresos de los ancianos insertados en el programa de atención domiciliaria que en los del grupo de comparación, en especial al servicio de urgencias con $70 \%$ de reingresos para el grupo de estudio contra 50\% para el grupo de comparación, lo cual tiene relación directa con la comorbilidad de los sujetos, la severidad de las enfermedades, así como con aspectos sociales identificados en otros estudios. En el caso del presente estudio, la comorbilidad de los sujetos del grupo de atención domiciliaria representó un punto de análisis importante, dado que se presentó una diferencia altamente significativa entre los grupos, especialmente por la alta comorbilidad del grupo de atención domiciliaria en $61.43 \%$ de los casos contra $36.66 \%$ de los sujetos del grupo de comparación, lo cual podría ser el factor de mayor peso para que en esta cohorte los reingresos 
no constituyeran un impacto a favor del programa, como lo expresan otros estudios. ${ }^{5,6,20,21}$ Asimismo, cabe mencionar que en un estudio relativo a la evaluación de este mismo programa'se evidenciaron resultados favorables al mismo en la contención de los reingresos hospitalarios y reducción de días estancia; la diferencia con este estudio previo podría atribuirse al estado de salud de los pacientes, toda vez que se consideraron pacientes que eran atendidos en el primer nivel de atención, mientras que en el presente estudio los pacientes fueron ingresados a hospitalización por presentar padecimientos agudos que, sumados a la alta comorbilidad prevaleciente, no permitieron evidenciar el impacto esperado en el programa ADEC, lo cual representa una limitación del estudio.

Ahora bien, acerca de la calidad de vida relacionada con la salud, la escala del SIP permitió retomar un concepto importante en la atención de los ancianos que es el impacto que tiene la enfermedad sobre su calidad de vida y, en este sentido, se constituye como una variable sensible a las acciones de un programa de atención domiciliaria. En relación con esta medición, los valores Proxy y las respuestas de los sujetos fueron homogéneas entre las dos mediciones, lo que permitió considerar los resultados de esta variable como un dato único de análisis, a pesar de haber considerado que la dimensión que obtendría un mayor cambio a favor del programa sería la dimensión física. Llama la atención el cambio en la dimensión psicosocial a favor del programa, un aspecto que vale la pena mencionar es que la medida global de calidad de vida no presentó diferencias entre los grupos, lo cual es un hallazgo consistente con investigaciones previas. En contraste, en la dimensión psicosocial de la calidad de vida relacionada con la salud, los estudios previos expresan que al analizar la calidad de vida según la enfermedad de base se observan diferencias en la respuesta de calidad de vida por dimensiones, como ocurrió en el estudio de Esbensen y colaboradores, ${ }^{22}$ donde, a partir de una cohorte de ancianos con cáncer terminal seguidos durante tres meses en un programa de atención por enfermería en domicilio, encontraron una percepción baja acerca de la red social y desconfianza por parte del paciente disminuyeron la percepción de la calidad de vida, así como también los niveles de dependencia física y económica con una diferencia significativa en relación con el grupo de comparación. Al relacionar estos resultados con el presente estudio, consideramos que a pesar de que la calidad de vida no se analizó por padecimientos, se podría suponer que al reforzar la red de apoyo mejoró la percepción del paciente respecto a la dimensión psicosocial.

Por otra parte, la sobrecarga de los cuidadores debe ser una variable a considerar ya que probablemente representa un impacto negativo para el grupo de atención domiciliaria, puesto que el programa seguramente no aporta elementos que disminuyan la sobrecarga de los cuidadores y sí aumenta las responsabilidades del mismo al soportar la estrategia de acción sobre el cuidador. Esta importante variable deberá ser considerada en estudios a futuro y constituirá un tema de enorme importancia dada la limitada respuesta del sistema de salud a las necesidades de cuidados a largo plazo que cada vez serán requeridos con mayor intensidad. De hecho, Schulz y colaboradores ${ }^{23}$ evaluaron una estrategia de atención domiciliaria de largo plazo para sujetos con demencia entre cuidadores en casa y cuidadores de sujetos institucionalizados; expresaron que los cuidadores en casa presentaron una mayor proporción estadísticamente significativa de ansiedad y depresión que los cuidadores del grupo de comparación, dato que se mantuvo al final del estudio.

La ventaja del presente estudio radicó en que los resultados y conclusiones generadas permiten a los tomadores de decisiones analizar los resultados del programa en las condiciones actuales en que se lleva a cabo, en especial aquéllas con deficiencias logísticas en cuanto a personal y otros recursos, para definir cambios a favor de la atención de los derechohabientes.

Es evidente que los resultados de esta investigación deben ser analizados considerando sus limitaciones. Evaluar el efecto de intervenciones con diseños no experimentales y no controlados puede derivar en evidencia débil debido a sesgos de selección, entre otros aspectos. Sin embargo, debe considerarse que el presente artículo hace referencia a un programa existente, es decir, a una evaluación de efectividad en circunstancias reales de operación.

\section{Conclusiones}

La introducción de modelos de atención domiciliaria representa una alternativa para la contención de servicios y para mejorar la red de apoyo en torno al anciano. Realizar estudios de costo efectividad probaría la pertinencia del programa dentro de las políticas públicas de atención a largo plazo.

\section{Agradecimientos}

Agradecemos al personal de los hospitales participantes por su colaboración para poder recabar la información de los participantes, en especial al equipo de trabajo del Programa de Atención Domiciliaria al Enfermo Crónico (ADEC). Este estudio fue financiado por el Fondo de Fomento a la Investigación del Instituto Mexicano del Seguro Social con el número FP-2005/1/I/001 y con 
recursos del proyecto "Evaluación del impacto de tres estrategias en la provisión de los Servicios de Salud a ancianos asegurados por el IMSS" financiado por CONACyT con el registro Salud-2004-C02/020.

Declaración de conflicto de intereses: Los autores declararon no tener conflicto de intereses.

\section{Referencias}

I. Douglas SL, Daly BJ, Kelley CG, O’Toole E, Montenegro H. Chronically Critically III Patients: Health-Related Quality of Life and Resource Use After a Disease Management Intervention. Am J Critical Care 2007:16:447-457.

2. Lynn J,Adamson DM. Adapting Health Care to Serious Chronic Illness in Old Age. California: RAND white papers, 2003: I-I9.

3. Baumgarten M, Lebel P, Laprise H, Leclerc Ch, Quinn Ch. Adult day care for the frail elderly: outcomes, satisfaction, and cost. J Aging Health 2002; 14:237-259.

4. Van Haastregt J, Diederiks JPM, van Rossum E, de Witte LP, Crebolder $\mathrm{H}$. Effects of preventive home visits to elderly people living in the community: systematic review. Brit Med Journal 2000;320:754-758. 5. Elkan R, Kendrick D, Dewey M, Hewitt M, Robinson J, Blair M, et al. Effectiveness of home based support for older people: systematic review and meta-analysis. Br Med J 200 I;323:719

6. Shepperd S, lliffe S. Hospital domiciliario versus atención hospitalaria estándar (Cochrane Review). En: La Biblioteca Cochrane Plus, 2008, Issue 3, CD000356

7. Hughes SL,Weaver FM, Giobbie-Hurder A, Manheim L, Henderson W, Kubal JD, et al. Effectiveness of team-managed home-based primary care: a randomized multicenter trial. JAMA 2000;284:2877-2885.

8. Sherwood S, Morris JN. The Pennsylvania domiciliary care experiment: I. Impact on quality of life.Am J Public Health 1983;73:646-653

9. Constantino P, Muñoz M, Garduño J. Evaluación económica del

Programa Atención Domiciliaria a pacientes con enfermedades crónicas y terminales. En: Duran L,Muñoz O (Eds). Retos de la seguridad social en salud en el siglo XXI: Evaluación y Gestión Tecnológica. México: IMSS, 2002:I| $3-\mid 22$.
10. Morales-Palomo T,Villareal-Ríos E. Impacto de la enseñanza a familiares de pacientes del programa Atención Domiciliaria al Enfermo Crónico ADEC. Revista de Salud Pública y Nutrición 2002;e(2).

II. Calderón A, Martínez LJ, Medrano RM,Vázquez M. Grado de dependencia y capacidades de auto cuidado del paciente adscrito al programa de atención domiciliaria al enfermo crónico (ADEC). Revista de la Facultad de Salud Pública y Nutrición de la Universidad de Nuevo León 2002:(2).

I2. Barrera C, Hernández C. Evaluación de la satisfacción de los pacientes pos operados de patología traumática y ortopédica de extremidad pélvica con atención médica domiciliaria versus consulta externa. Acta Ortopédica Mex 2004;18:33-36.

13. Programa de Atención Domiciliaria al Enfermo Crónico (ADEC). Documento institucional de Divulgación Interna. México: IMSS, 1990. 14. Reyes-Beaman S, Beaman PE, García-Peña C,Villa MA, Heres J, Cordova A, et al. Validation of a modified versión of the Minimental State Examination (MMSE) in Spanish. Aging Neuropsychol Cogn 2004; I I: I- I I. 15. Katz S, Ford AB, Moskowitz RW, Jacksion BA, Jaffe MW. Studies of Illness in the Aged. The index of ADL:A Standarized measure of Biological and Psychosocial Function. JAMA 1963; 185:94-99.

16. Bergner M, Bobbitt RA, Carter WB, Gilson BS. The Sickness Impact Profile: development and final revision of health status measure. Med Care 1981;19:787-805.

17. García-Peña C, Reyes H, Garduño J, Fajardo A, Martinez C. La calidad de vida en el paciente diabético tipo Il y factores relacionados. Rev Med IMSS 1995; 33:293-298.

18. Badia J,Alonso Y.Validity and Reproducibility of the Spanish Version of the Sickness Impact Profile.J Clin Epidemiol 1996;49:359-365.

19. Charlson ME, Pompei P,Ales KI, MacKenzie R.A new method of classifying prognostic comorbidity in longitudinal studies: development and validation.J Chronic Diseases 1987;40:373-383.

20. Smith A, Carusone S, Willison K, Babineau T, Smith S, Abernathy T, et al. Hospitalization and emergency department visits among seniors receiving homecare: a pilot study. BMC Geriatrics 2005;5(9).

21. Stuck A, Egger M, Hammer A, Minder C, Beck J. Home Visits to Prevent Nursing Home Admission and Functional Decline in Elderly People. JAMA 2002; 287: 1022-1028.

22. Esbensen BA, Osterlind K, Hallberg IR. Quality of life of elderly persons with cancer: a 3-month follow-up. Cancer Nurs 2006;29:2 I4-224. 23. Schulz R, Belle S, Czaja S, McGinnis K, Stevens A, Zhang S. Long-term Care Placement of Dementia Patients and Caregiver Health and Wellbeing. JAMA 2004;292:961-967. 